NBER WORKING PAPER SERIES

\title{
SMALL NOISE ASYMPTOTICS FOR A STOCHASTIC GROWTH MODEL
}

\author{
Noah Williams \\ Working Paper 10194 \\ http://www.nber.org/papers/w10194 \\ NATIONAL BUREAU OF ECONOMIC RESEARCH \\ 1050 Massachusetts Avenue \\ Cambridge, MA 02138 \\ December 2003
}

I thank William (Buz) Brock, Matthias Doepke, Han Hong, Ken Judd, Makoto Nirei, Simon Potter,Chris Sims, and seminar participants at Columbia University, Stanford University, the Federal Reserve Bank of New York, the 2002 Society for Economic Dynamics meetings, the 2003 Econometric Society Winter Meetings, the 2003 Society for Computational Economics Meetings, and the Conference on Learning and Bounded Rationality at the University of Illinois for helpful comments. Comments by an associate editor and an anonymous referee helped improve the paper substantially. Financial support from the National Science Foundation is gratefully acknowledged. The views expressed herein are those of the authors and not necessarily those of the National Bureau of Economic Research.

(C)2003 by Noah Williams. All rights reserved. Short sections of text, not to exceed two paragraphs, may be quoted without explicit permission provided that full credit, including (C) notice, is given to the source. 
Small Noise Asymptotics for a Stochastic Growth Model

Noah Williams

NBER Working Paper No. 10194

December 2003

JEL No. E32, C32

\begin{abstract}
We develop analytic asymptotic methods to characterize time series properties of nonlinear dynamic stochastic models. We focus on a stochastic growth model which is representative of the models underlying much of modern macroeconomics. Taking limits as the stochastic shocks become small, we derive a functional central limit theorem, a large deviation principle, and a moderate deviation principle. These allow us to calculate analytically the asymptotic distribution of the capital stock, and to obtain bounds on the probability that the log of the capital stock will differ from its deterministic steady state level by a given amount. This latter result can be applied to characterize the probability and frequency of large business cycles. We then illustrate our theoretical results through some simulations. We find that our results do a good job of characterizing the model economy, both in terms of its average behavior and its occasional large cyclical fluctuations.

Noah Williams

Department of Economics

Princeton University

Princeton, NJ 08544-1021

and NBER

noahw@princeton.edu
\end{abstract}




\section{INTRODUCTION}

Modern macroeconomics is built on the foundation of nonlinear dynamic stochastic general equilibrium (DSGE) models. In particular, the stochastic growth model is one of the most widely used models in all of economics, and is the standard model for business cycle analysis. However because the model can only be solved in closed form under very restrictive assumptions (such as log utility and full depreciation of capital), analysis of the model must resort to approximations. For example, a standard practice is to linearize the Euler equations which characterize the optimal solution around a deterministic steady state. ${ }^{1}$ In many cases, there is little discussion of the quality of such approximations, particularly for the stochastic properties of the economy. ${ }^{2}$ In this paper we provide some steps in this direction. We provide analytic asymptotic results which characterize the average behavior of the stochastic growth model and its occasional large fluctuations.

Our analysis considers limits as the standard deviation $(\sigma)$ of the stochastic technology shocks converges to zero. We show that the capital accumulation trajectories converge to the corresponding trajectories from a deterministic model. The limiting deterministic models are typically easier to analyze, particularly in the neighborhood of a steady state. The results provide analytic, theoretically justified approximations for stochastic models with small noise. There is less, if any, need for numerical methods and simulation. Further, the analytic expressions we obtain are useful for comparative statics and dynamics, and can potentially be used as a means for estimation. Interestingly, we find that many asymptotic properties of the economy can be described

\footnotetext{
${ }^{1}$ Papers which use linearizations are too numerous to list, but some notable applications in contexts similar to this paper include Magill (1977), Kydland and Prescott (1982), King, Plosser, and Rebelo (1988), and Cambpell (1994). A summary of a variety of numerical methods can be found in Judd (1998).

${ }^{2}$ An exception is the literature on perturbation methods, discussed in more detail below. A related issue, which we do not address is the approximation of welfare associated with linearized models. Kim and Kim (2003) have shown the potential problems this may cause.
} 
by a linear approximation. However for larger fluctuations, and to describe potential asymmetries in the time series, nonlinear methods are needed. While we focus on a relatively simple and standard model, the methods we develop can be applied to more general nonlinear DSGE models which may provide a closer match to the data.

In our analysis below, we obtain three different characterizations of the rate at which the stochastic model converges to the deterministic one. We first formulate a functional central limit theorem which shows that at rate $\sigma$, the centered capital trajectories are asymptotically normal. While this result holds for both for the level of capital and its logarithm, for our other results we consider solely the log of the capital stock. We then apply a large deviation principle, which shows that the absolute differences of the log capital trajectories converge to zero exponentially fast. We also obtain estimates of the average time it takes the log capital stock to differ from its steady state level by a given amount. Finally, we present a moderate deviation principle which provides similar results, but for an intermediate range of asymptotics between the functional central limit theorem and the large deviation principle.

Both the functional central limit theorem and the moderate deviation principle are expressed in terms of a linear approximation to the deterministic model. These results thus suggest that in order to consider the average behavior of the economy and even to consider some "extreme" events, a linear approximation is sufficient. However the linear approximation does not fully capture the large deviation properties, and it abstracts from certain asymmetries in the model due to nonlinearities. ${ }^{3}$ By applying the large deviation results, we show that the model economy is slightly asymmetric and is slightly more likely to exhibit recessions (appropriately defined) than booms.

This appears to be the first paper to apply these type of convergence results for the stochastic growth model. However there are several of related papers in the literature.

\footnotetext{
${ }^{3}$ There are higher order asymptotic results for continuous time diffusions in the literature, such as Fleming and Souganidis (1986) and Fleming and James (1992), which may capture some of these nonlinearities in an analytically tractable way. However to our knowledge there are no such results for our discrete time setting.
} 
In a continuous time setting, Prandini (1994) used the large deviation results of Azencott (1980) to analyze a stochastic Solow growth model. He showed that the capital trajectories in the stochastic model converges uniformly on a finite time horizon to the corresponding trajectories from the standard deterministic model. We obtain similar results in our section on large deviations. However, we provide a more detailed analysis with more explicit calculations, in addition to the wider array of asymptotic results we establish. Perhaps more importantly, our model incorporates explicit optimization, which broadens the potential scope of applications. However it also provides some complications in the analysis, as we must establish that the policy functions converge in an appropriate sense and satisfy some additional regularity conditions.

As we noted above, there is a closely related and extensive branch of the literature focusing on the properties of approximate solutions. However the functional approximation results do not characterize the stochastic properties of the model economy, which is our focus. In the mathematics literature, key papers include Fleming (1971) and Fleming and Souganidis (1986) who prove the uniform convergence of policy functions in continuous time models when the noise goes to zero. In the economics literature, an early contribution was Magill (1977) who derived an asymptotic linearquadratic approximation in a continuous time stochastic growth model. Judd (1998) contains a comprehensive overview of perturbation methods and their applications in economics. Judd and Guu $(1993,1997)$ present numerical methods based on Taylor expansions to analyze stochastic and deterministic growth models respectively. Gaspar and Judd (1997) provide high order expansions for multivariate models. Most of these papers focus on the local analytic properties of the solution, with a few providing global numerical results. But again, none of these touch on the issues we analyze.

\section{THE MODEL}

In this section we lay out the benchmark model for the analysis. It is a specialized Brock-Mirman (1972) economy with production, capital accumulation, and stochastic 
productivity growth. To simplify the presentation and analysis, we assume that technology shocks are permanent, which implies that the model has a single state variable. This assumption can be relaxed. Further, in the development of the paper it should be evident that our results have applications to more general nonlinear stochastic models.

\subsection{The Stochastic Growth Model}

We assume that output is produced according to a standard constant returns to scale Cobb-Douglas production function with parameter $\alpha$ :

$$
F(K, L)=K^{\alpha}(A L)^{1-\alpha}
$$

where $K$ is the capital stock, $L$ is the labor supply and $A$ is the labor-augmenting technology parameter. For simplicity, we fix the total labor supply at $L=1$. We assume that $A$ evolves exogenously as a unit root process in logarithms:

$$
\log A_{t+1}=\kappa+\log A_{t}+\sigma W_{t+1}
$$

where $W$ is a standard normal random variable and $\kappa \geq 0$ is the mean rate of technology growth. The unit root assumption is made for simplicity, and is roughly consistent with US time series data. Let $\delta$ be the depreciation rate of capital, and $C_{t}$ be consumption. Then the evolution equation for capital is given by:

$$
K_{t+1}=A_{t}^{1-\alpha} K_{t}^{\alpha}-C_{t}+(1-\delta) K_{t}
$$

Although the technological process is nonstationary, the ratios of capital to technology, $k_{t}=K_{t} / A_{t}$, and that of consumption to technology, $c_{t}=C_{t} / A_{t}$, are stationary. We therefore represent the problem in terms of the stationary variables. Normalizing by the technology level, (2) becomes:

$$
k_{t+1}=\theta Z_{t+1}^{\sigma}\left(k_{t}^{\alpha}-c_{t}+(1-\delta) k_{t}\right)
$$

where we define the lognormal random variable $Z^{\sigma}$ and constant $\theta$ as:

$$
Z_{t+1}^{\sigma}=\exp \left(-\sigma W_{t+1}\right), \quad \theta=\exp (-\kappa)
$$


A representative agent has time-additively separable preferences over consumption, with CRRA period utility:

$$
U(C)=\frac{C^{1-\gamma}}{1-\gamma}=A^{1-\gamma} \frac{c^{1-\gamma}}{1-\gamma} .
$$

The social planner's problem is to choose a consumption sequence to maximize the expected discounted utility of the representative agent. Thus we solve:

$$
\sup _{\left\{C_{t}\right\}} E \sum_{t=0}^{\infty} \beta^{t} U\left(C_{t}\right)
$$

subject to (2) and (1). Note that expressing utility in terms of $c_{t}$ makes the effective subjective discount factor $\beta\left(\theta Z_{t+1}^{\sigma}\right)^{\gamma-1}$, and thus introduces a form of preference shocks. Straightforward calculations, detailed in Appendix A, show that this Markov optimization problem has a solution which is a feedback control of the form: $c_{t}=c^{\sigma}\left(k_{t}\right)$. This implies that we can write the optimal capital evolution as:

$$
\begin{aligned}
k_{t+1} & =\theta Z_{t+1}^{\sigma}\left(k_{t}^{\alpha}-c^{\sigma}\left(k_{t}\right)+(1-\delta) k_{t}\right) \\
& \equiv \bar{f}^{\sigma}\left(Z_{t+1}^{\sigma}, k_{t} ; c^{\sigma}\right)
\end{aligned}
$$

where the notation in the second line emphasizes the dependence on the unknown consumption policy function $c^{\sigma}$. As is standard, this policy function satisfies the stochastic Euler equation, derived in Appendix A:

$$
c^{\sigma}(k)^{-\gamma}=\beta \int\left(\theta Z^{\sigma}\right)^{\gamma} c^{\sigma}\left(\bar{f}^{\sigma}\left(Z^{\sigma}, k ; c^{\sigma}\right)\right)^{-\gamma}\left[\alpha \bar{f}^{\sigma}\left(Z^{\sigma}, k ; c^{\sigma}\right)^{\alpha-1}+1-\delta\right] d G^{\sigma}\left(Z^{\sigma}\right)
$$

where $G^{\sigma}$ is the relevant lognormal distribution function.

In our analysis to follow, it helps to split the capital evolution into its conditional expectation and its martingale component. Therefore we define the expectation of the right side of (5), conditioned on $k_{t}=k$ as:

$$
f^{\sigma}(k)=\theta \exp \left(\frac{\sigma^{2}}{2}\right)\left(k^{\alpha}-c^{\sigma}(k)+(1-\delta) k\right)
$$


and the random component is then:

$$
\nu^{\sigma}(k)=\theta \exp \left(-\sigma W_{t+1}-\frac{\sigma^{2}}{2}\right)\left(k^{\alpha}-c^{\sigma}(k)+(1-\delta) k\right)
$$

Again, both of these depend on the unknown function $c^{\sigma}$, but we suppress this dependence in the notation. Thus we have an alternate expression for the capital evolution:

$$
k_{t+1}=f^{\sigma}\left(k_{t}\right)+\nu^{\sigma}\left(k_{t}\right)
$$

While the optimization problem is most naturally stated in terms of the level of the capital stock, for most of our analysis we will work with the logarithm of the capital stock. The multiplicative nature of the noise term in (5) makes the logs particularly easy to work with. Thus if we let $l_{t}=\log \left(k_{t}\right)$, we can take $\operatorname{logs}$ and rewrite:

$$
l_{t+1}=g^{\sigma}\left(l_{t}\right)-\sigma W_{t+1},
$$

where we define:

$$
g^{\sigma}(l)=\log \left[\exp (\alpha l)-c^{\sigma}(\exp (l))+(1-\delta) \exp (l)\right]-\kappa
$$

The conditional normality of $l_{t}$ greatly simplifies many of the results that follow.

\subsection{A Deterministic Growth Model}

Corresponding to the stochastic growth model, we can define a deterministic growth model by setting $\sigma=0$ in the equations above. This yields a discrete time version of a standard Ramsey-Cass-Koopmans model in which the technology grows at a constant rate. The implied evolution for capital is therefore:

$$
k_{t+1}=\theta\left[k_{t}^{\alpha}-c_{t}+(1-\delta) k_{t}\right]
$$

The optimal growth problem is then the deterministic analogue of (4), with law of motion (12). Again, straightforward calculations show that this problem has a feedback solution of the form $c_{t}=c^{0}\left(k_{t}\right)$. This implies that capital and log capital follow the 
deterministic difference equations:

$$
\begin{aligned}
k_{t+1} & =f^{0}\left(k_{t}\right), \\
l_{t+1} & =g^{0}\left(l_{t}\right),
\end{aligned}
$$

where $f^{0}$ and $g^{0}$ are obtained by replacing $c^{\sigma}$ with $c^{0}$ in (7) and (11), respectively. The optimal consumption policy satisfies the Euler equation analogous to (6):

$$
c^{0}(k)^{-\gamma}=\beta \theta^{\gamma} c^{0}\left(f^{0}\left(k ; c^{0}\right)\right)^{-\gamma}\left[\alpha f^{0}\left(k ; c^{0}\right)^{\alpha-1}+1-\delta\right] .
$$

\subsection{Comparisons of the Models}

Let $\left\{k_{t}^{\sigma}\right\}$ be a realization of the capital stock trajectory from the stochastic growth model, $\left\{k_{t}^{0}\right\}$ be the capital stock trajectory from the deterministic model, and $\left\{l_{t}^{\sigma}\right\}$ and $\left\{l_{t}^{0}\right\}$ their respective logarithms. As $\sigma \rightarrow 0$ we expect that, starting from the same initial value, the sample paths of the solution of the stochastic growth model would approach that of the deterministic model. In the rest of the paper we show that this is indeed the case, and we obtain explicit characterizations of the asymptotics at different rates of convergence. These results provide us with approximate characterizations of the stochastic economy when the noise is small.

Our results consider the normalized differences, for $\rho \geq 0$ :

$$
X_{t}^{\sigma, \rho}=\frac{1}{\sigma^{1-\rho}}\left(l_{t}^{\sigma}-l_{t}^{0}\right)
$$

In Section 3, we consider the case $\rho=0$, and present a functional central limit theorem (FCLT). We show that at rate $\sigma$, the normalized differences converge to a Gaussian linear autoregressive process. In Section 4 , we consider the case $\rho=1$, and formulate a large deviation principle (LDP). Thus we obtain bounds on the probability that (on a given finite horizon) the stochastic capital trajectory differs from the deterministic one by a given amount. We also consider the exit problem, which provides estimates of how long it typically takes for the log capital stock to depart from its steady state level by a given amount. In Section 5 , we consider a range of cases where $0<\rho<1$, 
and apply a so-called moderate deviation principle (MDP). These results are similar to the large deviation principle, but for $\sigma$-dependent neighborhoods. This modification leads to explicit results. In Section 6, we illustrate our results through some explicit calculations and simulations in a calibrated model. Our results show that the theoretical predictions provide a good explanation of the behavior of the model as observed in simulations. We also illustrate some of the differences between the large deviation and moderate deviation results, which have implications for linear solution methods. Finally, Section 7 concludes. Throughout we make smoothness assumptions on the consumption policies, and we also assume that the stochastic policies converge to the deterministic one. In Appendix A we formally establish these results. Appendices B and $\mathrm{C}$ collect proofs of some of the results in the text.

\section{A FUNCTIONAL CENTRAL LIMIT THEOREM}

In this section we begin our analysis of the stochastic growth model. We present a functional central limit, which follows Klebaner and Nerman (1994), and shows that the normalized differences $X_{t}^{\sigma, 0}$ from (16) converge to a Gaussian linear autoregression. We also show that a similar result holds for the capital levels. As noted above, in order to make clear which features of the model are required for each result we make direct assumptions on the policy functions. These assumptions are verified in our application in Appendix. Because this model has been so widely studied, some of the smoothness and stability conditions we require follow from known results in the literature. We also require that the policy functions converge, and this assumption requires the most argument. We establish it by building up from properties of the value functions.

To cover both logs and levels, in the assumptions we use $h(x)$ as a stand-in for either $g(l)$ or $f(k)$, and we always use a subscript $x$ for a derivative of a function. Klebaner and Nerman (1994) impose boundedness conditions on the derivatives of policy functions which cause some slight complications in our analysis. Due to the Inada condition on the utility function, the slopes of the policy functions (in levels) 
increase to infinity at zero capital. However on any compact set bounded away from zero, the boundedness conditions are satisfied. Therefore we extend the results by truncating the state evolution to a compact set, but relaxing the truncation in the limit. In what follows, we require $x \in \mathcal{X}$ with $\mathcal{X}$ a compact set. This notation is a stand in for $l \in \mathcal{L} \subset \mathbb{R}$ and $k \in \mathcal{K} \subset \mathbb{R}_{++}$with $\mathcal{L}$ and $\mathcal{K}$ compact.

Assumption 3.1. On any compact set $\mathcal{X}$, the function $h^{\sigma}$ is continuous, twice continuously differentiable, and has bounded derivatives $h_{x}^{\sigma}$ and $h_{x x}^{\sigma}$ for all $\sigma \geq 0$.

Assumption 3.2. On any compact set $\mathcal{X}, h^{\sigma} \rightarrow h^{0}$ uniformly as $\sigma \rightarrow 0$.

Assumption 3.3. On a compact set $\mathcal{X}, h^{0}$ has a unique fixed point $x^{*}$ which is stable, i.e. $\left|h_{x}^{0}\left(x^{*}\right)\right|<1$, and whose domain of attraction includes all of $\mathcal{X}$.

Theorem 3.1. Suppose that Assumptions 3.1 and 3.2 hold for $h=g$. Then as $\sigma \rightarrow 0$, the normalized differences $\left\{X_{t}^{\sigma, 0}\right\}$ converge weakly to a process $\left\{X_{t}\right\}$. The limit process follows the linear Gaussian autoregression, dependent on the deterministic process $\left\{l_{t}^{0}\right\}$ :

$$
X_{t+1}=g_{x}^{0}\left(l_{t}^{0}\right) X_{t}+B_{t+1}
$$

where $\left\{B_{t+1}\right\}$ is an i.i.d. sequence of standard normal random variables.

Proof. See Appendix B.

Although we focus on the case of log-normal technology shocks $Z_{t}^{\sigma}$, Theorem 3.1 can be extended to a more general setting, although not as general as a typical central limit theorem. The theorem would continue to hold for any distribution which converges to 
a normal with small noise, i.e. for which:

$$
\frac{\log \left(Z_{t}^{\sigma}\right)}{\sigma} \Rightarrow N(0,1) \text { as } \sigma \rightarrow 0
$$

Obvious examples include a Student's $t$ distribution or a multinomial approximation to a normal, where in each case we increase the precision of the approximation as we shrink the standard deviation. Of more direct importance, for small $\sigma$ a lognormal distribution also converges weakly to a normal (see Johnson and Kotz, 1970). This underlies our results on the limit distribution of the capital stock levels $k_{t}$ below.

Since there is a unique steady state of the deterministic model, Theorem 3.1 implies that the differences from the steady state are asymptotically normal. As this result has implications for approximate solution methods, we state it explicitly.

Corollary 3.1. Suppose that Assumptions 3.1-3.3 hold for $h=g$. Then as $\sigma \rightarrow 0$ and $t \rightarrow \infty$ the following holds asymptotically:

$$
l_{t+1}^{\sigma}-l^{*}=g_{x}^{0}\left(l^{*}\right)\left(l_{t}^{\sigma}-l^{*}\right)+\sigma B_{t+1} .
$$

Therefore, as $\sigma \rightarrow 0$ and $t \rightarrow \infty,\left\{l_{t}^{\sigma}\right\}$ converges to a stationary Gaussian process with mean $l^{*}$ and variance $\frac{\sigma^{2}}{1-g_{x}^{0}\left(l^{*}\right)^{2}}$.

This result shows that the log capital stock asymptotically follows a Gaussian linear autoregression centered on the deterministic steady state. One simple application of this result concerns the calibration of models. Typically, models are calibrated to match the first moments of key time series. However Corollary 3.1 allows the use of analytic asymptotic second moments as well. In particular, it provides a means of calibrating the risk aversion parameter $(\gamma)$ by using the (asymptotic) autocorrelation of the capital stock, which equals the derivative of the policy function at the steady state. Notice that this calibration is completely analytic, and does not require simulation of the model. We use this result to calibrate the model in Section 6 below. 
For the case of the levels, we substitute $k_{t}$ for $l_{t}$ in our definition of $X_{t}^{\sigma, 0}$. Then, making the appropriate substitutions, we have the following results.

TheOrem 3.2. Suppose that Assumptions 3.1 and 3.2 hold for $h=f$. Then as $\sigma \rightarrow 0$, the normalized differences converge weakly to the process:

$$
X_{t+1}=f_{x}^{0}\left(k_{t}^{0}\right) X_{t}+f^{0}\left(k_{t}^{0}\right) B_{t+1}
$$

Suppose in addition that Assumption 3.3 holds for $h=f$. Then the results of Corollary 3.1 hold with $\sigma f^{0}\left(k^{*}\right)$ in place of $\sigma$ and the other obvious substitutions.

\section{A LARGE DEVIATION PRINCIPLE}

In the previous section, we showed as the technology shock standard deviation $\sigma$ goes to zero, the log capital stock from the stochastic growth model converges to the deterministic one and the normalized differences are asymptotically normal. In this section we consider the asymptotics of the differences, without normalizing by $\sigma$. We provide a large deviation principle (LDP) which shows that, on a given finite horizon, the differences converge to zero exponentially fast. We use this to analyze episodes where the log capital stock differs from its steady state value by a given amount. We show that the time between such events increases exponentially, and we provide an estimate of the typical length. We also show that the curvature of the policy function determines whether large increases or decreases are more likely. In Section 6 below we illustrate the implications of these results for business cycles. The results in this section follow Klebaner and Zeitouni (1994), with our proofs in Appendix C.1.

We now define some terminology. Let a sequence $\left\{Z^{\epsilon}\right\}$ be defined on a probability space $(\Omega, \mathcal{F}, P)$ and taking values in a Polish space $\mathcal{X}$. A rate function $S: \mathcal{X} \rightarrow[0, \infty]$ has the property that for any $M<\infty$ the level set $\{x \in \mathcal{X}: S(x) \leq M\}$ is compact. 
Definition 4.1. A sequence $\left\{Z^{\epsilon}\right\}$ satisfies a large deviation principle on $\mathcal{X}$ with rate function $S$ and speed $\epsilon$ if the following two conditions hold.

1. For each closed subset $F$ of $\mathcal{X}, Z^{\epsilon}$ satisfies the large deviation upper bound:

$$
\limsup _{\epsilon \rightarrow 0} \epsilon \log P\left\{Z^{\epsilon} \in F\right\} \leq-\inf _{x \in F} S(x) .
$$

2. For each open subset $G$ of $\mathcal{X}, Z^{\epsilon}$ satisfies the large deviation lower bound:

$$
\liminf _{\epsilon \rightarrow 0} \epsilon \log P\left\{Z^{\epsilon} \in G\right\} \geq-\inf _{x \in G} S(x) .
$$

If the limit of the sequence is not $F$ and $G$, the probability the sequence enters these sets converges to zero. A LDP insures that the convergence is exponential, with the leading exponent determined by the rate function. The definitions also show how in large deviation theory the evaluation of a probabilistic statements is characterized by an optimization problem. This makes the theory amenable for analysis and leads to natural solution methods to apply it in practice.

We now develop a LDP for finite time paths of the log capital stock, considered as elements of the product space $\mathcal{L}^{T} \subset \mathbb{R}^{T}$. We equip the space with Euclidean metric, denoted $d_{T}$, and let $[u]_{T}=\left(u_{0}, u_{1}, \ldots u_{T}\right)$. Then we have the following result.

Theorem 4.1. Suppose that Assumptions 3.1 and 3.2 hold for $h=g$. Then on a given finite horizon $[0, T]$ the sequence $\left\{l_{t}^{\sigma}\right\}$ satisfies a large deviation principle on $\left(\mathcal{L}^{T}, d_{T}\right)$ with speed $\sigma^{2}$ and rate function:

$$
S\left(T,[u]_{T}\right)=\frac{1}{2} \sum_{t=0}^{T-1}\left(u_{t+1}-g^{0}\left(u_{t}\right)\right)^{2} .
$$

Thus Theorem 4.1 shows that the probability the stochastic and deterministic paths differ by a given amount converges to zero exponentially fast, and determines the rate of convergence. The rate function for the time paths is the cumulation of each one-step transition. Since each step is conditionally normal, the rate function is quadratic. The 
theorem also implies that once the capital stock reaches its deterministic steady state level, it has a small probability of exiting a neighborhood of the steady state.

We now spell out this implication of the LDP by considering what is known as the exit or escape problem. To begin with this analysis, we define:

$$
V(x, y)=\inf _{\left\{[u]_{T}: u_{0}=x, u_{T}=y, T<\infty\right\}} S\left(T,[u]_{T}\right) .
$$

This gives the minimized "cost" of moving from $x$ to $y$ in some finite horizon, as evaluated by the rate function $S$. A path $[u]_{T}$ which achieves the minimum clearly exists and is called a dominant escape path. We focus mainly on escapes from the deterministic steady state, so we define:

$$
\bar{V}(\epsilon)=\inf _{\left\{y:\left|y-l^{*}\right| \geq \epsilon\right\}} V\left(l^{*}, y\right) .
$$

Let $Y^{*}$ be the set of minimizers in $(20)$ and define the exit time from the interval as:

$$
\tau^{\sigma}(\epsilon)=\inf \left\{t>0:\left|l_{t}^{\sigma}-l^{*}\right| \geq \epsilon,\left|l_{0}^{\sigma}-l^{*}\right|<\epsilon\right\}
$$

We now show that the exit times increase to infinity exponentially fast at a given rate, and we determine the end of the interval where an exit will most likely take place.

Theorem 4.2. Suppose that Assumptions 3.1-3.3 hold with $h=g$. Then given $\epsilon>0$, for any $\eta>0$ we have:

$$
\lim _{\sigma \rightarrow 0} P\left(\exp \left(\frac{\bar{V}(\epsilon)-\eta}{\sigma^{2}}\right) \leq \tau^{\sigma}(\epsilon) \leq \exp \left(\frac{\bar{V}(\epsilon)+\eta}{\sigma^{2}}\right)\right)=1 .
$$

For any exit which occurs at $l_{\tau^{\sigma}}^{\sigma}$ and for any $\eta>0$ there exists a $y^{*} \in Y^{*}$ such that:

$$
\lim _{\sigma \rightarrow 0} P\left(\left|l_{\tau^{\sigma}}^{\sigma}-y^{*}\right|<\eta\right)=1
$$

To apply these results, we must solve the minimization problem in (19)-(20), which does not generally have an explicit solution. In Section 6 we solve the problem numerically, while we now provide a local characterization. We show that for small escapes, 
the curvature of the policy function determines whether a positive or negative escape is more likely. Loosely speaking, with a convex (log) capital accumulation function, the proportion of income saved increases with $l$ and hence there is a slower return to $l^{*}$ following a positive shock. The formal result applies a perturbation argument, as detailed in Appendix C.2.

Theorem 4.3. Suppose that Assumptions 3.1-3.3 hold for $h=g$. In a neighborhood of the stable equilibrium $l^{*}$ the rate function in (19) has the expansion:

$$
V\left(l^{*}, y\right)=\frac{\left(l^{*}-y\right)^{2}}{2}\left(1-g_{x}^{0}\left(l^{*}\right)^{2}\right)-\frac{\left(l^{*}-y\right)^{3}}{2} g_{x x}^{0}\left(l^{*}\right) g_{x}^{0}\left(l^{*}\right)^{2} \frac{1-g_{x}^{0}\left(l^{*}\right)^{2}}{1-g_{x}^{0}\left(l^{*}\right)^{3}}+O\left(\left|l^{*}-y\right|^{4}\right)
$$

Thus if $g^{0}$ is strictly convex (respectively, strictly concave), an exit from the interval $\left(l^{*}-\epsilon, l^{*}+\epsilon\right)$ happens at $l^{*}+\epsilon$ (respectively, $\left.l^{*}-\epsilon\right)$ with probability converging to one as $\sigma \rightarrow 0$ and $\epsilon \rightarrow 0$. If $g^{0}$ is linear the exit is equally likely to happen at either endpoint.

\section{A MODERATE DEVIATION PRINCIPLE}

In this section we derive some asymptotic results which in effect combine the insights of the two previous sections. We present a moderate deviation principle (MDP), which considers the sequence $X_{t}^{\sigma, \rho}$ for the intermediate case $0<\rho<1$, whereas we have previously considered $\rho=0$ and $\rho=1$. In essence, a moderate deviation principle is a large deviation principle for the normalized process over a slower range of speeds. Our results in this section provide simple, explicit asymptotic characterizations.

Following Klebaner and Liptser (1999), we first provide some of the heuristic arguments which convey the main ideas. For $\rho \in(0,1)$ we can write $X_{t}^{\sigma, \rho}=\sigma^{\rho} X_{t}^{\sigma, 0}$. Now by Theorem 3.1, we know that $X_{t}^{\sigma, 0} \Rightarrow X_{t}$ where $\left\{X_{t}\right\}$ is the Gaussian linear autoregression in (17). Now suppose that the processes $\left\{X_{t}^{\sigma, \rho}\right\}$ and $\left\{\tilde{X}_{t}^{\sigma, \rho}\right\}=\left\{\sigma^{\rho} X_{t}\right\}$ 
satisfy the same large deviation principle. ${ }^{4}$ Recall from (17) that $\left\{\widetilde{X}_{t}^{\sigma, \rho}\right\}$ satisfies:

$$
\widetilde{X}_{t+1}^{\sigma, \rho}=g_{x}^{0}\left(l_{t}^{0}\right) \tilde{X}_{t}^{\sigma, \rho}+\sigma^{\rho} B_{t+1}
$$

Then relying on Theorem 4.1, we could show that on an infinite horizon the sequence $\left\{\widetilde{X}_{t}^{\sigma, \rho}\right\}$ satisfies a large deviation principle with speed $\sigma^{2 \rho}$ and rate function:

$$
J([u])=\frac{1}{2} \sum_{t=0}^{\infty}\left(u_{t+1}-g_{x}^{0}\left(l_{t}^{0}\right) u_{t}\right)^{2}
$$

where and $[u]=\left(u_{0}, u_{1}, \ldots\right)$. The rate function $J$ is quadratic in $u$, as it relies on the central limit results, and this allows for explicit analytic characterization.

We now verify that these heuristics do in fact hold. The results here are the parallel of Theorems 4.1 and 4.2, with proofs also in Appendix C.1. We focus on the infinite horizon MDP, which implies a corresponding MDP over any finite horizon. We thus extend the state space to $\mathcal{L}^{\infty}$ and equip it with the metric:

$$
d([u],[v])=\sum_{t=0}^{\infty} 2^{-t-1} \frac{\left|u_{t}-v_{t}\right|}{1+\left|u_{t}-v_{t}\right|} .
$$

We now define the exit time for the normalized process:

$$
\tau^{\sigma, \rho}(\epsilon)=\inf \left\{t>0:\left|X_{t}^{\sigma, \rho}\right| \geq \epsilon\right\}
$$

This is the first time the process $\left\{l_{t}^{\sigma}\right\}$ exits from an window of width $2 \epsilon \sigma^{1-\rho}$ centered on the path $\left\{l_{t}^{0}\right\}$. The MDP exit sets thus shrink with $\sigma$, unlike the LDP. Then we have the following results, providing the MDP and a characterization of the exit problem.

TheOREM 5.1. Let $0<\rho<1$, and suppose Assumptions 3.1-3.2 hold for $h=g$.

1. Then the sequence $\left\{X_{t}^{\sigma, \rho}\right\}$ satisfies a moderate deviation principle on the infinite product space $\left(\mathcal{L}^{\infty}, d\right)$ with speed $\sigma^{2 \rho}$ and rate function $J$ given in (21).

2. Suppose that Assumptions 3.3 holds as well, and let $l_{0}^{0}=l^{*}$. Then we have:

$$
\lim _{\sigma \rightarrow 0} \sigma^{2 \rho} \log E \tau^{\sigma, \rho}(\epsilon)=\frac{\epsilon^{2}}{2}\left(1-g_{x}^{0}\left(l^{*}\right)^{2}\right) \equiv \bar{J}(\epsilon) .
$$

\footnotetext{
${ }^{4}$ As emphasized by Kushner (1984), this will not hold in general, as there is only weak convergence.
} 


\section{AN APPLICATION}

In this section we illustrate our theoretical results in a calibrated model. We choose the parameters of the model so that it matches certain features of US time series data. To calibrate the process for $A_{t}$, we used data on the cumulative Solow residual from the DRI database, following the construction of Stock and Watson (1999). ${ }^{5}$ The mean growth rate $\kappa$ and standard deviation $\sigma$ of the $A_{t}$ process are chosen to match the implied Solow technology process at an annual frequency.

TABLE 1.

The baseline model parameters.

\begin{tabular}{ccc}
\hline Parameter & Description & Annual Value \\
\hline$\kappa$ & technology growth & 0.0176 \\
$\sigma$ & technology shock standard deviation & 0.0492 \\
$\alpha$ & labor's share & 0.65 \\
$\delta$ & depreciation & 0.0517 \\
$\beta$ & subjective discount factor & 0.9847 \\
$\gamma$ & relative risk aversion & 4 \\
\hline
\end{tabular}

The remaining parameters are chosen in accordance with our theoretical predictions. From our Corollary 3.1 we predict that for small $\sigma$ the mean of the log capital stock should be $l^{*}$ and its autocorrelation $g_{x}^{0}\left(l^{*}\right)$. In Appendix A we provide analytic expressions for these variables in terms of the model parameters. From our data sample, we find that the log capital stock has mean 1.396 and annual autocorrelation of

\footnotetext{
${ }^{5}$ The data are quarterly from 1959:Q1 to 1999:Q2 and are constructed from output (GDP less farm, housing and government), capital (interpolation of annual values of fixed non-residential capital stock using quarterly investment), and labor (hours of employees on non-agricultural payrolls). We then construct the technology shock process using a labor's share value of $\alpha=0.65$.
} 


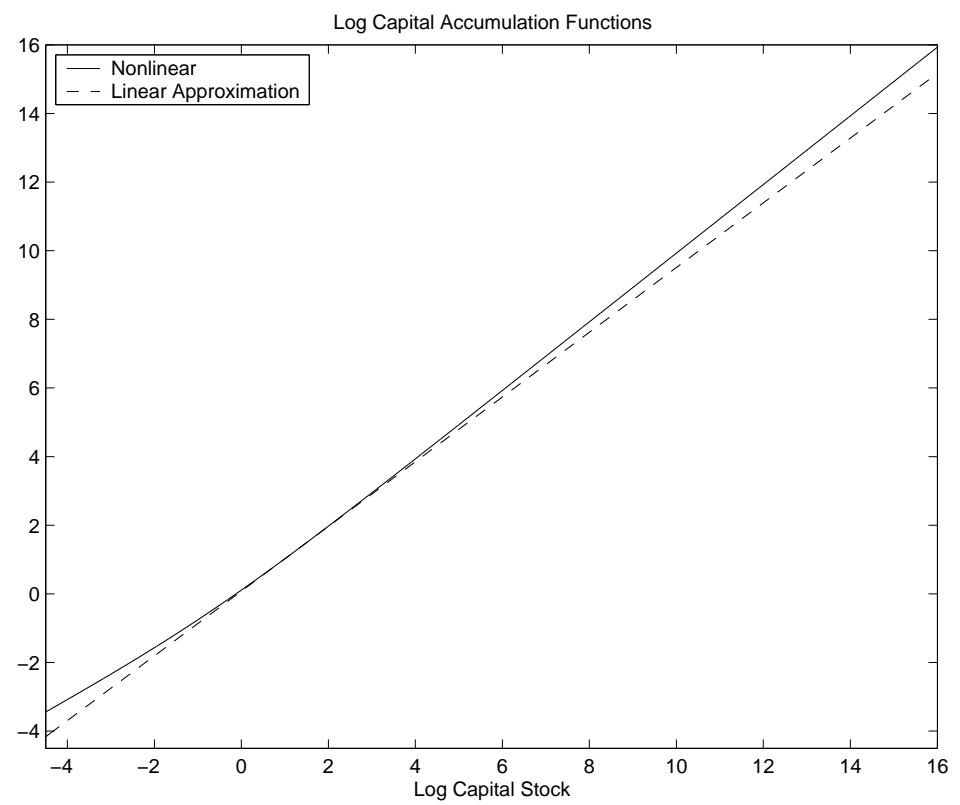

FIGURE 1. The log capital accumulation function from the numerical solution of the deterministic growth model and its linear approximation.

0.943. Given the values of $\alpha$ and $\kappa$ above, for any choice of the relative risk aversion parameter $\gamma$ the expressions for $\left(l^{*}, g_{x}^{0}\left(l^{*}\right)\right)$ determine pairs $(\beta, \delta)$ which are consistent with the data. However for arbitrary $\gamma$, we are not guaranteed that $\beta$ and $\delta$ will be between zero and one. By experimenting, we found that setting $\gamma$ to the plausible, if slightly high, value of 4 led to reasonable results for the subjective discount rate and deprecation. Our specific parameter choices are summarized in Table 1.

To provide a basis of comparison for our theoretical results, we numerically solve for the policy functions. Appendix C.3 describes our solution method. In Figure 1 we plot the log capital accumulation function from the deterministic model along with its linear approximation. Here we see that the policy function is nearly linear, with a slight convexity which is apparent far from the steady state. This provides a preview of some of the results to follow: the linear approximation provides a good characterization of the policy function except far from the steady state. Furthermore by Theorem 4.3 , the slight convexity suggests that positive escapes will be more likely. 


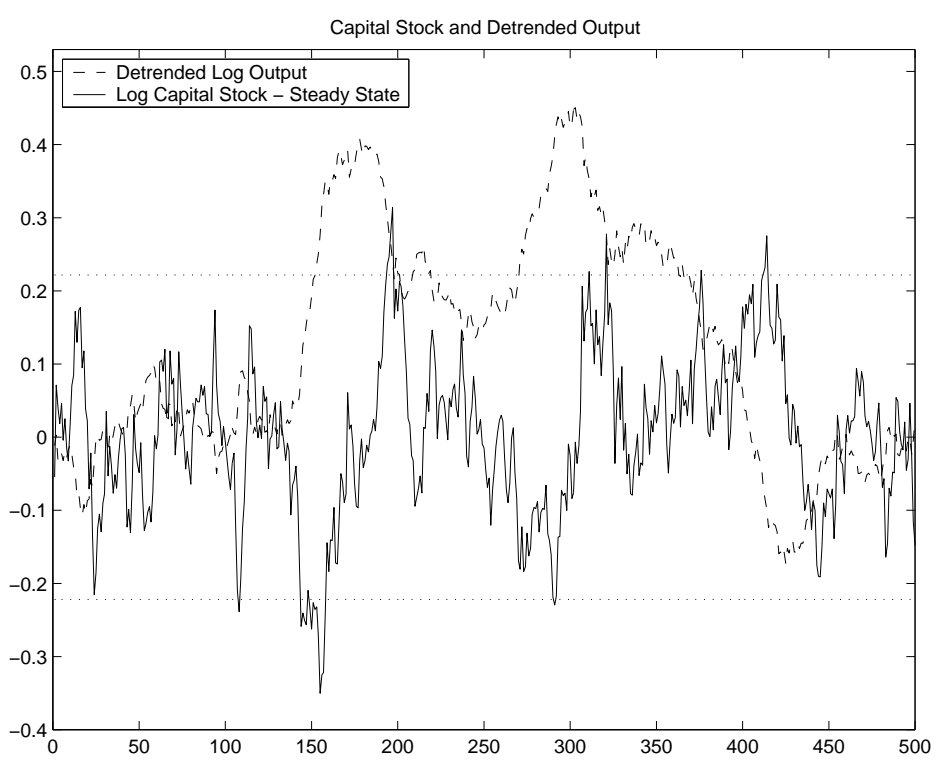

FIGURE 2. A simulation of the logarithm of the capital stock and detrended output. The dashed lines show the large deviation bands.

We will identify large movements in the log capital stock with business cycles, which is justified in Figure 2. The figure plots simulated data from the baseline parameterization, showing the value of the log capital stock minus its deterministic steady state level and the detrended log output. The figure clearly shows the negative correlation between the log capital stock and log output, with large changes in output corresponding to the large changes of opposite direction in the capital stock. The dotted lines in the figure plot the escape sets that we consider below. Notice that the negative escapes correspond to booms (peaks of output relative to trend), while positive escapes correspond to recessions (falls in output relative to trend).

We now illustrate our asymptotic characterizations. Table 2 summarizes the simulation results for different levels of the standard deviation of the technology shock process, which we index by multiples of our benchmark $\sigma$ from Table 1 . Turning first to the functional central limit theorem predictions, the first two columns of the table give the autocorrelation and standard deviation of the log capital stock $l_{t}$ from simulations of 5000 periods. Corollary 3.1 above provides theoretical predictions for the mean (1.396), autocorrelation (0.943), and the standard deviation of the series (0.148), 
TABLE 2.

Simulation results for different levels of the technology shock standard deviation.

\begin{tabular}{|c|c|c|c|c|c|c|c|}
\hline \multirow{2}{*}{$\begin{array}{l}\text { Tech. } \\
\text { Std. }\end{array}$} & \multicolumn{3}{|c|}{ FCLT } & \multicolumn{2}{|c|}{$\mathrm{LDP}$} & \multicolumn{2}{|c|}{ MDP } \\
\hline & Mean & Auto. & Std. & Mean & Positive & Mean & Positive \\
\hline Dev. & Level & Corr. & Dev. & Time & (Percent) & Time & (Percent) \\
\hline $2 \sigma$ & 1.32 & 0.946 & 0.301 & 10.1 & 49.6 & 18.9 & 49.8 \\
\hline$\sigma$ & 1.37 & 0.947 & 0.152 & 42.3 & 51.6 & 42.3 & 51.6 \\
\hline $0.75 \sigma$ & 1.38 & 0.947 & 0.114 & 108.3 & 52.8 & 65.5 & 53.5 \\
\hline $0.5 \sigma$ & 1.38 & 0.947 & 0.076 & 1008.1 & 58.2 & 132.0 & 53.1 \\
\hline
\end{tabular}

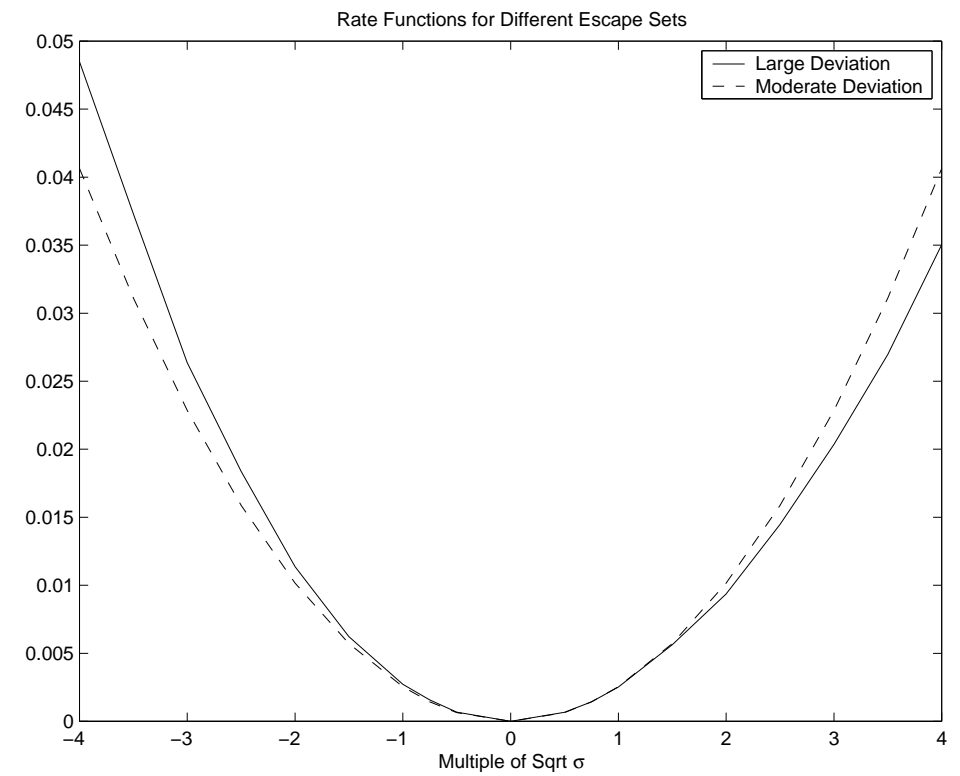

FIGURE 3. Comparisons of the rate functions for the large deviation principle and the moderate deviation principle, for varying escape sets (indexed by $\epsilon=n \sqrt{\sigma}$, for different $n$ ).

which formed the basis of our calibration. For the benchmark $\sigma$, the predictions are very close to the simulated values in the table. Further, the standard deviation decreases proportionately to $\sigma$ in the simulations, just as the theory predicts.

We next apply the LDP and MDP to characterize rare events in the log capital stock process. In both cases, the probability of an escape and the mean escape times are characterized by the rate function. For the MDP, Theorem 5.1 provides this explicitly, 

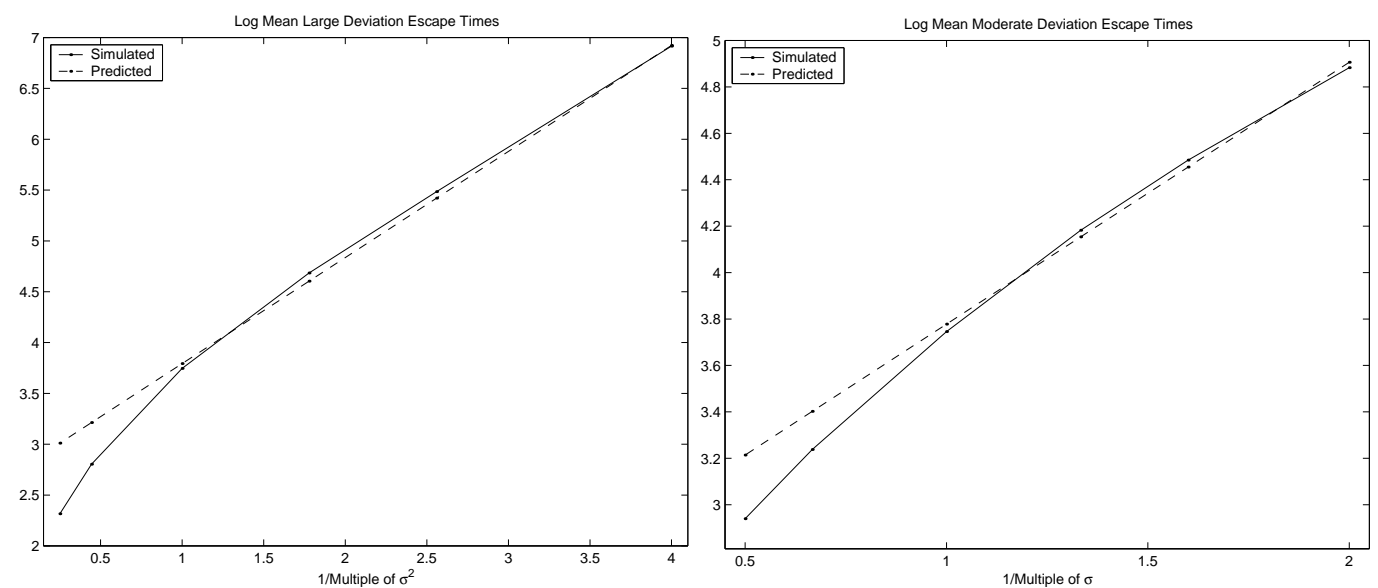

FIGURE 4. Logarithm of mean escape times for different standard deviations. The solid lines plot the results from simulations, while the dashed lines plot predicted results. The left panel plots the results for the large deviation principle, and the right panel plots the moderate deviation principle.

while for the LDP we must use numerical methods, as described in Appendix C.3. The rate functions $\bar{V}$ and $\bar{J}$ for the LDP and MDP are shown in Figure 3 for different values of the escape set $\epsilon$. We know that the MDP rate function is symmetric, while the nonlinearity of the policy function causes the LDP rate function to be slightly asymmetric. For small escape sets, the two are similar, as the policy function is wellapproximated by a linear function over much of the state space. Therefore even for the analysis of some rare events, the linear approximation may provide acceptable results. However, just as the linear approximation breaks down far from the steady state, for large escapes the rate functions differ substantially. Due to the convexity of the policy function, the LDP rate function is lower for positive escapes, which confirms the local analysis of Theorem 4.3. But even the small differences for small escape sets matter, as they lead to differences in the exponential increase in the time between escapes. Therefore, for small $\sigma$ both large booms and large recessions become increasingly unlikely, but we expect recessions to occur more frequently than booms.

We now illustrate these results through simulations. The last four columns of Table 2 provide a summary of 5000 simulated escape paths for different levels of the technology shock. For each simulation run, we initialize the log capital stock at the 
steady state and simulate until either a positive or negative escape happens. ${ }^{6}$ We let the size of the escape set be fixed at $\epsilon=0.222$, corresponding to a nearly 16 percent change in the log capital stock, a sizable fluctuation. For the MDP, we let $\rho=0.5$. Recall that the MDP escape sets shrink with $\sigma$, and thus escapes are more likely in this case. The table clearly shows the rapid increase in the mean escape times in both cases. The asymmetry is also evident, especially in the LDP results. At the baseline $\sigma$, we find that recessions account for 52 percent of the escapes. When $\sigma$ is cut in half, this increases to 58 percent of the LDP escapes (and over 53 percent for the MDP). For small noise the asymmetry of the large business cycle fluctuations becomes more apparent, and as predicted we find recessions to be more likely than booms.

Our results also give accurate predictions of the rate of increase in escape times. For the LDP, Theorem 4.2 shows that for small $\sigma$ the escape times are approximately:

$$
\log E \tau^{d \sigma} \approx \log C_{L}+\bar{V} / \sigma^{2}
$$

for some constant $C_{L}$. Similarly, for the MDP with $\rho=0.5$, Theorem 5.1 shows that for small $\sigma$ the mean escape times are approximately:

$$
\log E \tau^{d \sigma, 0.5} \approx \log C_{M}+\bar{J} / \sigma
$$

for some constant $C_{M}$. Thus the escape times increase exponentially with the inverse of standard deviation for the MDP and the inverse of the variance for the LDP, with the rate of increase determined by the rate function. This is shown graphically in Figure 4, where we plot the log of the mean escape times from Table 2 along with our predictions. We choose the constants $C_{L}$ and $C_{M}$ in order to get a good fit, as our

\footnotetext{
${ }^{6}$ As $\sigma$ goes to zero, $l_{t}$ converges to $l^{*}$, the fixed point of $g^{0}$. But for $\sigma>0$ the fixed point of $g^{\sigma}$ is different from $l^{*}$. In our simulations, we thus look at escapes from the fixed point instead of from $l^{*}$. This has essentially no effect on the mean escape times, but it does affect the results on the asymmetry of escapes. Note that in Table 2 the mean of $l_{t}^{\sigma}$ converges to $l^{*}$ from below. By analyzing escapes from $l^{*}$ we would distort the results in favor of negative escapes.
} 
results only determine the slopes of the lines. The figure shows that our predictions accurately characterize the rate of increase in the mean escape times. Further, both the LDP and MDP predictions are much closer for small $\sigma$, as our theory predicts.

\section{CONCLUSION}

In this paper we have presented and applied a variety of asymptotic methods which characterize the properties of the stochastic growth model. We have shown that as the standard deviation of the technology shock process gets small, the log capital stock process converges to a Gaussian linear autoregression. Further, we have characterized the probability and frequency of large fluctuations in the log capital stock. Our results have shown that for small noise, the capital stock process converges to the deterministic steady state, and that the autocorrelation and variance of the log capital stock process can be characterized analytically. Additionally we have shown that for small noise large booms and recessions become increasingly unlikely, although recessions are more likely than booms. Finally, we showed that the average time between business cycles increased exponentially, and provided accurate predictions of the rate of increase.

For several reasons, in this paper we have focused on the stochastic growth model. It is one of the most widely used models, and is a standard test case for new methods. Further, the fact that there was a single state variable provided substantial technical simplifications. However it should be clear that our results apply much more broadly, and could be extended to multidimensional cases. Of particular interest may be our implications for linearization methods. We show that for small noise, a linear approximation provides a characterization of the average behavior of the model and also certain occasional large fluctuations. As linearization remains one of the most common solution methods, these results have many potential applications. 


\section{APPENDIX A}

\section{Properties of the Policy Functions}

As described previously it is convenient to scale consumption and capital by the technology level. This allows us to derive Bellman equations that do not depend on $A$, but only on $k$, thus simplifying our analysis. As is well-known, the Bellman equation for the original problem is:

$$
v(K, A)=\max _{C}\left\{\frac{C^{1-\gamma}}{1-\gamma}+\beta \int v\left(K^{\prime}, A^{\prime}\right) d G(Z)\right\} \text { s.t. (1), (2) }
$$

where $G$ is the log-normal distribution function. We use the normalizations $k=K / A$ and $c=C / A$, then verify that the value functions satisfy $v(K, A)=A^{1-\gamma} v(k)$. This leads to the reduced Bellman equation:

$$
v(k)=\max _{c}\left\{\frac{c^{1-\gamma}}{1-\gamma}+\beta \int(\theta Z)^{\gamma-1} v\left(k^{\prime}\right) d G(Z)\right\} \text { s.t. (3). }
$$

The utility function satisfies the Inada conditions, so that we know solutions must be interior. The first order condition and an application of the envelope theorem then yield the Euler equation (6) in the text. Analogous results in the deterministic case lead to the Euler equation (15).

Standard results, as in Stokey, Lucas, and Precott (1989) show that in both the deterministic and stochastic cases the policy functions $k^{\prime}=f^{0}(k)$ and $k^{\prime}=f^{\sigma}(k)$ are continuous and bounded for finite $k$. Simple arguments also show that the policy functions are strictly increasing, and further that the consumption policies are also strictly increasing. Results from Araujo (1991) and Santos (1991) show that the policy functions are continuously differentiable in the deterministic case, and Santos (1991) provides a bound on the derivative. By adapting results from Amir (1997), we also have that the policy functions are twice continuously differentiable in the stochastic case (see also Blume et al, 1982), and are bounded on the interior of the state space. Finally, the results in Santos (1993) suggest that in our model the policy function in the deterministic case is also twice continuously differentiable. As an optimal path is always interior and, as we show below, there is a unique interior stable steady state, we can apply the imply the implicit function theorem to establish the higher order smoothness of the policy function.

We next turn to deriving analytic expressions for the deterministic steady state $l^{*}$ and the derivative of the policy function at the steady state $g_{x}^{0}\left(l^{*}\right)$. These expressions form the basis of our calibrations. From the Euler equation (15), the unique interior deterministic steady state is easily seen to be:

$$
k^{*}=\left(\frac{1-\beta \theta^{\gamma}+\delta \beta \theta^{\gamma}}{\alpha \beta \theta^{\gamma}}\right)^{\frac{1}{\alpha-1}} .
$$


Taking logs gives $l^{*}$. Then from (3), we find the steady state consumption $c^{*}=\left(k^{*}\right)^{\alpha}+\frac{\theta-1-\delta \theta}{\theta} k^{*}$. This in turn allows us to evaluate the derivatives of the utility function at the steady state: $u^{\prime}=\left(c^{*}\right)^{-\gamma}$, and $u^{\prime \prime}=$ $-\gamma\left(c^{*}\right)^{-\gamma-1}$. Following Judd and Guu (1997), we apply the implicit function theorem to the Euler equation (15) to find an expression for the derivative of the consumption function at the steady state $c^{\prime}=c_{x}^{0}\left(k^{*}\right)$ :

$$
\left(c^{\prime}\right)^{2}\left[\beta \theta^{\gamma+1} u^{\prime \prime}\left(\alpha\left(k^{*}\right)^{\alpha-1}+1-\delta\right)\right]+c^{\prime}\left[u^{\prime \prime}-\beta \theta^{\gamma+1} u^{\prime \prime}\left(\alpha\left(k^{*}\right)^{\alpha-1}+1-\delta\right)^{2}\right]-\beta \theta^{\gamma} u^{\prime} \alpha(\alpha-1)\left(k^{*}\right)^{\alpha-2}=0
$$

Using the expression for $k^{*}$, this can be reduced to:

$$
\begin{aligned}
\left(c^{\prime}\right)^{2}+D_{1} c^{\prime}+D_{2} & =0, \text { with : } \\
D_{1}=\frac{1}{\theta}-\frac{1}{\beta \theta^{\gamma}}, D_{2} & =-\beta \theta^{\gamma+1} \frac{c^{*}}{\gamma} \alpha(1-\alpha)\left(k^{*}\right)^{\alpha-2} .
\end{aligned}
$$

Since $D_{2}<0$, this insures that $c^{\prime}$ is real, and since we know $c^{\prime}$ is positive, we have that:

$$
c^{\prime}=-D_{1}+\sqrt{D_{1}^{2}-4 D_{2}}>-D_{1}
$$

With this expression, we can then evaluate $g_{x}^{0}\left(l^{*}\right)$ :

$$
g_{x}^{0}\left(l^{*}\right)=\frac{\alpha\left(k^{*}\right)^{\alpha}-k^{*} c^{\prime}+(1-\delta) k^{*}}{\left(k^{*}\right)^{\alpha}-c^{*}+(1-\delta) k^{*}} .
$$

As $f^{0}$ is strictly increasing, $g_{x}^{0}=k f_{x}^{0} / f^{0}$ is strictly positive. Using the expressions for $c^{\prime}, c^{*}$, and $k^{*}$ we have:

$$
\begin{aligned}
c^{\prime}>-D_{1} & =\frac{1}{\theta}-\frac{1}{\beta \theta^{\gamma}}=\alpha\left(k^{*}\right)^{\alpha-1}+1-\delta-\frac{1}{\theta} \\
& =\frac{c^{*}}{k^{*}}-(1-\alpha)\left(k^{*}\right)^{\alpha-1}
\end{aligned}
$$

and therefore $g_{x}^{0}\left(l^{*}\right)<1$, so that the unique interior steady state is stable.

All that remains to be shown is the convergence of the stochastic policy function to the deterministic one. As above, we confine our attention to a compact set $\mathcal{K} \subset(0, \infty)$. Our proof adapts results in Santos and Vigo (1998). First note that the sequence $\left\{Z^{\sigma}\right\}$ converges weakly to 1 as $\sigma \rightarrow 0$. Next consider the deterministic value function $v^{0}(k)$, and note that $\left(Z^{\sigma}\right)^{\gamma-1} v^{0}\left(Z^{\sigma} x\right)$ is continuous and uniformly integrable (since $v^{0}$ is bounded above). Therefore:

$$
\lim _{\sigma \rightarrow 0} \int\left(Z^{\sigma}\right)^{\gamma-1} v^{0}\left(Z^{\sigma} x\right) d G^{\sigma}\left(Z^{\sigma}\right)=v^{0}(x)
$$

Furthermore, since $v(K, A)$ is a concave function for all $\sigma \geq 0,\left(Z^{\sigma}\right)^{\gamma-1} v^{0}\left(Z^{\sigma} x\right) \leq v^{0}(x)$, and therefore the convergence in (A.2) is monotone, and therefore by Dini's Theorem (see Dudley, 1989) uniform on compact sets. Next, we define the Bellman operator on the right side of (A.1) as $T^{\sigma}$, with the corresponding operator 
$T^{0}$ in the deterministic case. By our uniform convergence result, we then have that $T^{\sigma} v^{0}(k) \rightarrow T^{0} v^{0}(k)$. Additionally, since $T^{\sigma} v^{0}(k) \leq T^{0} v^{0}(k)=v^{0}(k)$, this convergence is again uniform on compact sets. Finally, letting $\|\cdot\|$ be the sup norm on the compact set $\mathcal{K}$, we have:

$$
\begin{aligned}
\left\|v^{0}-v^{\sigma}\right\| & =\left\|T^{0} v^{0}-T^{\sigma} v^{\sigma}\right\| \\
& \leq\left\|T^{0} v^{0}-T^{\sigma} v^{0}\right\|+\left\|T^{\sigma} v^{0}-T^{\sigma} v^{\sigma}\right\| \\
& \leq\left\|T^{0} v^{0}-T^{\sigma} v^{0}\right\|+\beta \theta^{\gamma-1}\left\|v^{0}-v^{\sigma}\right\|
\end{aligned}
$$

by the triangle inequality and the contraction property of $T^{\sigma}$. Thus:

$$
\left\|v^{0}-v^{\sigma}\right\| \leq \frac{1}{1-\beta \theta^{\gamma-1}}\left\|T^{0} v^{0}-T^{\sigma} v^{0}\right\|
$$

and so $v^{\sigma}$ converges uniformly to $v^{0}$, which implies that $c^{\sigma}$ converges pointwise to $c^{0}$. This in turn implies the pointwise convergence of $g^{\sigma}$ to $g^{0}$.

To show that the convergence of the policy functions is uniform, for a given $k$ define the function:

$$
G(c)=\frac{c^{1-\gamma}}{1-\gamma}+\beta \theta^{\gamma-1} v^{0}\left(\theta\left(k^{\alpha}-c+(1-\delta) k\right)\right) .
$$

Let $c^{0}=c^{0}(k)$ and $c^{\sigma}=c^{\sigma}(k)$ and note that $G\left(c^{0}\right)=v^{0}(k)$ and $G\left(c^{\sigma}\right) \geq T^{\sigma} v^{0}(k)$ so that $G\left(c^{0}\right)-G\left(c^{\sigma}\right) \leq$ $T^{0} v^{0}-T^{\sigma} v^{0}$. Then note that $G^{\prime}\left(c^{0}\right)=0$ and $G(c)$ is concave with a bounded second derivative on $\mathcal{K}$ (since the utility and value functions have finite second derivatives for $k>0$ ). Call the bound $\eta$. Then we have:

$$
\frac{\eta}{2}\left(c^{0}-c^{\sigma}\right)^{2} \leq G\left(c^{0}\right)-G\left(c^{\sigma}\right)
$$

and therefore:

$$
\left|c^{0}-c^{\sigma}\right| \leq \sqrt{\frac{\eta}{2}\left|T^{0} v^{0}-T^{\sigma} v^{0}\right|} .
$$

Since this holds for all $k$, we can take the sup over $k$, and the uniform convergence thus follows.

Moreover the convergence of the value functions on $\mathcal{K}$ is exponential at rate $\sigma^{2}$, implying that the convergence of the policy functions is exponential at rate $\sigma$. This is evident from the following:

$$
\begin{aligned}
\left\|T^{\sigma} v^{0}-T^{0} v^{0}\right\| & =\left\|\max _{c}\left\{\frac{c^{1-\gamma}}{1-\gamma}+\beta \int(\theta Z)^{\gamma-1} v^{0}\left(k^{\prime}\right) d G(Z)\right\}-\max _{c}\left\{\frac{c^{1-\gamma}}{1-\gamma}+\beta \theta^{\gamma-1} v^{0}\left(k^{\prime}\right)\right\}\right\| \\
& \leq\left\|\beta \theta^{\gamma-1} \max _{k^{\prime}}\left\{\int(\theta Z)^{\gamma-1} v^{0}\left(k^{\prime}\right) d G(Z)-v^{0}\left(k^{\prime}\right)\right\}\right\| \\
& \leq\left\|\beta \theta^{\gamma-1} \max _{x}\left\{\int Z^{\gamma-1} v^{0}(Z x) d G(Z)-v^{0}(x)\right\}\right\| \\
& \leq\left\|\beta \theta^{\gamma-1} \max _{x} v_{x}^{0}(x) x E[\exp ((\gamma-1) \sigma W)-\exp (\gamma \sigma W)]\right\| \\
& =\left|\beta \theta^{\gamma-1} \max _{x} v_{x}^{0}(x) x\left[\exp \left(\frac{1}{2}(1-\gamma)^{2} \sigma^{2}\right)-\exp \left(\frac{1}{2} \gamma^{2} \sigma^{2}\right)\right]\right| .
\end{aligned}
$$


Here the third line uses the definition of $k^{\prime}$ in the stochastic model, the fourth uses the concavity of $v^{0}$, the boundedness of the derivatives of $v^{0}$ on $\mathcal{K}$, and the definition of $Z$, and the fifth uses the properties of lognormal random variables. On the compact set $\mathcal{K}$ the capital stock and the derivative of the value function are both bounded, yielding the result.

\section{APPENDIX B}

\section{Functional Central Limit Theorem}

In this appendix, we prove Theorem 3.1 by extending the results of Klebaner and Nerman (1994). The basis of our result is their Theorem 3, which requires that Assumption 3.1 hold globally, not just on restrictions to compact sets. We use a truncation argument following Kushner and Yin (1997) in order to extend the result and show that the truncation is not needed in the limit. As in most of the paper, we focus on the results in logarithms, i.e. for $l=\log k$. The results for the levels are similar, and follow from the following preliminary result, which follows simply from the definitions of the functions and L'Hopital's rule.

LEMMA B.1. Let Assumptions 3.1 and 3.2 hold for $h=f$. Then for each fixed $k$, we have:

$$
\lim _{\sigma \rightarrow 0} \frac{1}{\sigma^{2}} E \nu^{\sigma}(k)^{2}=f^{0}(k)^{2}
$$

In this section, since we focus on the case $\rho=0$ we suppress the second superscript and write $X_{t}^{\sigma}=X_{t}^{\sigma, 0}$. First we truncate the process. That is, for every integer $M$ let $q_{M}$ be a continuous function on the line satisfying: $-M \leq q_{M}(x) \leq M, q^{M}(x)=x$ for $|x| \leq M, q^{M}(x)=M$ for $x \geq M+1$, and $q^{M}(x)=-M$ for $x \leq-(M+1)$. Note that $q_{x}^{M}(x)=1$ for $|x| \leq M$ and 0 for $|x| \geq M+1$. Then let $l_{0}^{\sigma, M}=l_{0}^{\sigma}$ and for $t \geq 0$ define the truncated process:

$$
l_{t+1}^{\sigma, M}=g^{\sigma}\left(q^{M}\left(l_{t}^{\sigma, M}\right)\right)-\sigma W_{t+1} .
$$

Then we define the normalized differences: $X_{t}^{\sigma, M}=\frac{l_{t}^{\sigma, M}-l_{t}^{0}}{\sigma}$. Note that we do not need to truncate $l_{t}^{0}$ as long as $\left|l_{0}^{0}\right|<M$, which we assume. Under our assumptions, the truncated process (B.1) satisfies the conditions of Klebaner and Nerman (1994), Theorem 3. Since the truncation is not applied to the deterministic process, we have that truncated normalized differences $\left\{X_{t}^{\sigma, M}\right\}$ converge to weakly to the autoregression $\left\{X_{t}\right\}$ in $(17)$. 
We now show that the truncation is unnecessary in the limit by establishing the tightness of the untruncated normalized process. The results of Klebaner and Nerman (1994) establish weak convergence in the product space, so it is enough to verify tightness at each date. Thus we need to show that for any $t$ we have:

$$
\lim _{K \rightarrow \infty} \sup _{\sigma} P\left(\left|X_{t}^{\sigma}\right| \geq K\right)=0
$$

By Prohorov's Theorem (see Kushner and Yin (1997), Theorem 7.3.1), tightness implies the existence of a weakly converging subsequence. As the truncation is not applied in the limit, the weak limit of the untruncated process must be the same as the truncated process.

Notice that from any initial condition $x \in \mathcal{L}$, we have:

$$
X_{1}^{\sigma}=\frac{l_{1}^{\sigma}-l_{1}^{0}}{\sigma}=\frac{g^{\sigma}(x)-g^{0}(x)}{\sigma}+W_{1} .
$$

The tightness of this sequence thus follows by the tightness of the normal random variable $W_{1}$. Then we proceed by induction. Thus for any $t$ we have:

$$
\begin{aligned}
X_{t+1}^{\sigma, 0} & =\frac{l_{t+1}^{\sigma}-l_{t+1}^{0}}{\sigma}=\frac{g^{\sigma}\left(l_{t}^{\sigma}\right)-\sigma W_{t+1}-g^{0}\left(l_{t}^{0}\right)}{\sigma} \\
& =\frac{1}{\sigma}\left[g^{\sigma}\left(l_{t}^{\sigma}\right)-g^{0}\left(l_{t}^{\sigma}\right)+g^{0}\left(l_{t}^{\sigma}\right)-g^{0}\left(l_{t}^{0}\right)\right]-W_{t+1} .
\end{aligned}
$$

Therefore we have:

$$
P\left(\left|X_{t}^{\sigma}\right| \geq K\right) \leq P\left(\left|g^{\sigma}\left(l_{t}^{\sigma}\right)-g^{0}\left(l_{t}^{\sigma}\right)\right| \geq K \sigma\right)+P\left(\left|g^{0}\left(l_{t}^{\sigma}\right)-g^{0}\left(l_{t}^{0}\right)\right| \geq K \sigma\right)+P\left(\left|W_{t+1}\right| \geq K \sigma\right)
$$

By the tightness of the normal distribution, the third term goes to zero with $K$. Then notice that we have:

$$
\left|g^{0}\left(l_{t}^{\sigma}\right)-g^{0}\left(l_{t}^{0}\right)\right|=\left|g_{x}^{0}\left(l_{t}^{0}\right)\right|\left|l_{t}^{\sigma}-l_{t}^{0}\right|+O\left(\left|l_{t}^{\sigma}-l_{t}^{0}\right|^{2}\right)
$$

Since $x=l_{0}^{0} \leq l_{t}^{0} \leq l^{*}$ the derivative is bounded and by the induction hypothesis $\left|l_{t}^{\sigma}-l_{t}^{0}\right|$ converges to zero with $\sigma$, and the second term is of order $\sigma^{2}$. This implies that the second term in (B.2) converges to zero at rate $\sigma$, which in turn implies that it goes to zero with $K$. Finally, we split the first term in (B.2) depending on whether $l_{t}^{\sigma}$ is in $\mathcal{L}$. Notice that:

$$
\begin{aligned}
P\left(\left|g^{\sigma}\left(l_{t}^{\sigma}\right)-g^{0}\left(l_{t}^{\sigma}\right)\right| \geq K\right) & =P\left(\left|g^{\sigma}\left(l_{t}^{\sigma}\right)-g^{0}\left(l_{t}^{\sigma}\right)\right| \geq K \| l_{t}^{\sigma} \in \mathcal{L}\right) P\left(l_{t}^{\sigma} \in \mathcal{L}\right) \\
& +P\left(\left|g^{\sigma}\left(l_{t}^{\sigma}\right)-g^{0}\left(l_{t}^{\sigma}\right)\right| \geq K \| l_{t}^{\sigma} \in \mathcal{L}^{c}\right) P\left(l_{t}^{\sigma} \in \mathcal{L}^{c}\right) .
\end{aligned}
$$

Notice that by the LDP results in Section 4, the probability of being in $\mathcal{L}$ at any date $t$ is of order 1 as $\sigma \rightarrow 0$ and the probability of being in $\mathcal{L}^{c}$ goes to zero (exponentially fast) with $\sigma^{2}$. Further, we have previously 
established that on $\mathcal{L}$ the policy functions converge uniformly at rate $\sigma$. Thus the fist term on the right of (B.3) converges to zero at rate $\sigma$. To bound the term on $\mathcal{L}^{c}$ notice that $\lim _{k \rightarrow 0} f^{\sigma}(k) / f^{0}(k)=1$ and $\lim _{k \rightarrow \infty} f^{\sigma}(k)=H k$ for some $H>0$ for all $\sigma \geq 0$. Thus $\left|g^{\sigma}(k)-g^{0}(k)\right|$ is bounded on $\mathcal{L}^{c}$. Putting this together implies that both terms on the right of (B.3) converge to zero at rate $\sigma$, and thus the first term in (B.2) converges to zero with $K$. This completes the proof of tightness, and so the result follows.

\section{APPENDIX C}

\section{Additional Proofs and Calculations}

\section{C.1. PROOFS OF LDP AND MDP RESUlTS}

We begin with an intermediate result on the one-step transitions of the log capital stock as in (10). We define $l_{x}^{\sigma}$ as the random variable with distribution identical to $l_{t+1}$ conditioned on $l_{t}=x$ :

$$
l_{x}^{\sigma} \sim N\left(g^{\sigma}(x), \sigma^{2}\right)
$$

LEMMA C.1. Suppose that Assumptions 3.1 and 3.2 hold for $h=g$. Then for any given finite $x,\left\{l_{x}^{\sigma}\right\}$ satisfies a large deviation principle on $\mathcal{L} \subset \mathbb{R}$ with speed $\sigma^{2}$ and rate function:

$$
I(x, y)=\frac{1}{2}\left(y-g^{0}(x)\right)^{2}
$$

This one-step LDP is a building block for the results to follow.

Proof. The result follows from the Gärtner-Ellis Theorem (see Dembo and Zeitouni (1998), Theorem 2.3.6). To apply the theorem, we need to calculate the limiting logarithmic moment generating function:

$$
H(x, \lambda)=\lim _{\sigma \rightarrow 0} \sigma^{2} \log E \exp \left(\frac{\lambda l_{x}^{\sigma}}{\sigma^{2}}\right)=\lambda g^{0}(x)+\frac{\lambda^{2}}{2},
$$

where the second equality follows from Assumption 3.2. Then the rate function is given by the Legendre transform of $H$ :

$$
I(x, y)=\sup _{\lambda \in \mathbb{R}}[\lambda y-H(x, \lambda)]
$$

which gives the result.

Proof (Theorem 4.1). Follows from Klebaner and Zeitouni (1994), Lemma 2.1. The necessary conditions of their theorem are easily verified given our assumptions and the form of the rate function $I$ established in 
Lemma C.1. In particular, their Lemma 2.5 holds under Assumption 3.2 and implies the uniformity of the large deviation principle. The continuity of $I$ and the other technical conditions follow as in the proof of their Theo-

rem 3.1 under our Assumptions 3.1 and 3.2.

Proof (Theorem 4.2). Follows from Klebaner and Zeitouni (1994), Theorem 2.1 and the remark which follows it. The necessary conditions are shown to hold using the same arguments as in our Theorem 4.1.

Proof (Theorem 5.1). Part 1 follows from Klebaner and Liptser (1999), Theorem 2. Their necessary conditions are easily verified given our assumptions and the form of the $H$ function defined in (C.2). Under our assumptions, Part 2 follows from Part 1 and Klebaner and Liptser (1999), Theorem 4.

\section{C.2. LOCAL EXPANSION OF THE LDP RATE FUNCTION}

In this appendix we derive the local expansion in Theorem 4.3. The idea adapts a continuous time result of Kasa (2001), but the derivation here is rather different and more involved. We expand the function $V(y) \equiv V\left(l^{*}, y\right)$ around $y=l^{*}$. At this point the optimal path is $u_{t}=l^{*}$ for all $t$. For a given $y$ reached at date $T$, each step $u_{t}$ of the optimal path will be an implicit function of $y$. However an application of the envelope theorem to problem (C.15) gives:

$$
\begin{aligned}
\frac{d V}{d y} & =y-g^{0}\left(u_{T-1}\right) \\
\frac{d^{2} V}{d y^{2}} & =1-g_{x}^{0}\left(u_{T-1}\right) \frac{d u_{T-1}}{d y} \\
\frac{d^{3} V}{d y^{3}} & =-g_{x}^{0}\left(u_{T-1}\right) \frac{d^{2} u_{T-1}}{d y^{2}}-g_{x x}^{0}\left(u_{T-1}\right)\left(\frac{d u_{T-1}}{d y}\right)^{2} .
\end{aligned}
$$

Note that the first derivative (C.3) equals zero when evaluated at $y=u_{T-1}=l^{*}$.

To evaluate the higher order derivatives, we use the first order conditions from problem (C.15):

$$
u_{t}-g^{0}\left(u_{t-1}\right)-\left(u_{t+1}-g^{0}\left(u_{t}\right)\right) g_{x}^{0}\left(u_{t}\right)=0
$$

for $t=1, \ldots, T-1$. Differentiating (C.6) gives implicit expressions for $\frac{d u_{t}}{d y}$. When evaluated at $l^{*}$ this gives:

$$
\left(1+g_{x}^{0}\left(l^{*}\right)^{2}\right) \frac{d u_{t}}{d y}-g_{x}^{0}\left(l^{*}\right) \frac{d u_{t-1}}{d y}-g_{x}^{0}\left(l^{*}\right) \frac{d u_{t+1}}{d y}=0
$$

with boundary conditions $\frac{d u_{0}}{d y}=0$ and $\frac{d u_{T}}{d y}=1$. For use below, note that (C.7) is a homogenous second order difference equation, whose characteristic equation has roots $g_{x}^{0}\left(l^{*}\right)$ and $1 / g_{x}^{0}\left(l^{*}\right)$. Therefore the general 
solution can be written:

$$
\frac{d u_{t}}{d y}=c_{1} g_{x}^{0}\left(l^{*}\right)^{T-t}+c_{2} g_{x}^{0}\left(l^{*}\right)^{t-T}
$$

for some $c_{1}, c_{2}$. To satisfy the boundary conditions (for $T \rightarrow \infty$ ) we must have $c_{1}=1$ and $c_{2}=0$, so that

$$
\frac{d u_{t}}{d y}=g_{x}^{0}\left(l^{*}\right)^{T-t}
$$

Substituting this expression into (C.4) gives:

$$
\left.\frac{d^{2} V}{d y^{2}}\right|_{l^{*}}=1-g_{x}^{0}\left(l^{*}\right)^{2}
$$

Note that this agrees with the expressions in Lewis (1986), who on pp. 32-35 explicitly solves this problem with a linear state evolution. This follows since a problem with linear evolution whose slope is $g_{x}^{0}\left(l^{*}\right)$ yields the identical difference equation (C.7).

Using similar logic, we evaluate the second derivative with respect to the end point $y$ by implicitly differentiating (C.6) again. After simplifying and evaluating at $l^{*}$ we obtain:

$$
\begin{aligned}
\left(1+g_{x}^{0}\left(l^{*}\right)^{2}\right) \frac{d^{2} u_{t}}{d y^{2}} & -g_{x}^{0}\left(l^{*}\right) \frac{d^{2} u_{t-1}}{d y^{2}}-g_{x}^{0}\left(l^{*}\right) \frac{d^{2} u_{t+1}}{d y^{2}} \\
& +3 g_{x}^{0}\left(l^{*}\right) g_{x x}^{0}\left(l^{*}\right)\left(\frac{d u_{t}}{d y}\right)^{2}-g_{x x}^{0}\left(l^{*}\right)\left(2 \frac{d u_{t}}{d y} \frac{d u_{t+1}}{d y}+\left(\frac{d u_{t-1}}{d y}\right)^{2}\right)=0
\end{aligned}
$$

Using (C.8), this simplifies to:

$$
\left(1+g_{x}^{0}\left(l^{*}\right)^{2}\right) \frac{d^{2} u_{t}}{d y^{2}}-g_{x}^{0}\left(l^{*}\right) \frac{d^{2} u_{t-1}}{d y^{2}}-g_{x}^{0}\left(l^{*}\right) \frac{d^{2} u_{t+1}}{d y^{2}}+g_{x x}^{0}\left(l^{*}\right) g_{x}^{0}\left(l^{*}\right)^{2(T-t)-1}\left(3 g_{x}^{0}\left(l^{*}\right)^{2}-2-g_{x}^{0}\left(l^{*}\right)^{3}\right)=0,
$$

with boundary conditions $\frac{d^{2} u_{0}}{d y^{2}}=\frac{d^{2} u_{T}}{d y^{2}}=0$. Thus (C.10) is a nonhomogeneous equation of the same form as (C.7). We transform it to a homogeneous equation by defining the variable $z_{t}=\frac{d^{2} u_{t}}{d y^{2}}-\mu_{t}$, where

$$
\mu_{t}=\frac{g_{x x}^{0}\left(l^{*}\right)\left(3 g_{x}^{0}\left(l^{*}\right)^{2}-2-g_{x}^{0}\left(l^{*}\right)^{3}\right)}{g_{x}^{0}\left(l^{*}\right)^{4}-g_{x}^{0}\left(l^{*}\right)^{3}-g_{x}^{0}\left(l^{*}\right)+1} g_{x}^{0}\left(l^{*}\right)^{2(T-t)} .
$$

Then $z_{t}$ follows the same difference equation as (C.7), but now with boundary conditions $z_{0}=-\mu_{0}=0$, $z_{T}=-\mu_{T}$. This means that we have the solution:

$$
z_{t}=-\mu_{T} g_{x}^{0}\left(l^{*}\right)^{T-t}
$$

and therefore:

$$
\frac{d^{2} u_{t}}{d y^{2}}=z_{t}+\mu_{t}=\mu_{T}\left(g_{x}^{0}\left(l^{*}\right)^{2(T-t)}-g_{x}^{0}\left(l^{*}\right)^{T-t}\right)
$$

Evaluating (C.12) at $T-1$ and using (C.11) gives:

$$
\frac{d^{2} u_{T-1}}{d y^{2}}=\frac{g_{x x}^{0}\left(l^{*}\right) g_{x}^{0}\left(l^{*}\right)\left(3 g_{x}^{0}\left(l^{*}\right)^{2}-2-g_{x}^{0}\left(l^{*}\right)^{3}\right)}{g_{x}^{0}\left(l^{*}\right)^{4}-g_{x}^{0}\left(l^{*}\right)^{3}-g_{x}^{0}\left(l^{*}\right)+1}\left(g_{x}^{0}\left(l^{*}\right)-1\right)
$$


Then substituting (C.8) and (C.13) into (C.5) and simplifying gives:

$$
\left.\frac{d^{3} V}{d y^{3}}\right|_{l^{*}}=-3 g_{x x}^{0}\left(l^{*}\right) g_{x}^{0}\left(l^{*}\right)^{2} \frac{1-g_{x}^{0}\left(l^{*}\right)^{2}}{1-g_{x}^{0}\left(l^{*}\right)^{3}} .
$$

Collecting (C.9) and (C.14) along with Taylor's theorem gives the expansion stated in Theorem 4.3. The conclusions follow from Theorem 4.2, where we note that by Assumption 3.3 the sign of the third derivative term is determined by $g_{x x}^{0}$.

\section{C.3. NUMERICAL METHODS}

To find the policy functions numerically, we solve the Euler equations (6) and (15) on a grid of 15,001 points centered on the steady state level, using log-linear interpolation. For the stochastic model, we approximate the conditional expectation using Gauss-Hermite quadrature with 51 nodes. We also apply numerical methods to solve for the dominant escape path for the LDP. Using (C.1) we can re-state the minimization problem as:

$$
\inf _{\left\{T<\infty,[u]_{T}\right\}} \frac{1}{2} \sum_{t=0}^{T-1}\left(u_{t+1}-g^{0}\left(u_{t}\right)\right)^{2}
$$

subject to $u_{0}=l^{*}, u_{T}=l^{*} \pm \epsilon$. Our solution strategy works with the first order conditions. For any given horizon $T$, problem (C.15) is a two-point boundary value problem for a path $[u]_{T}$ which starts at $l^{*}$ and ends at $l^{*} \pm \epsilon$ at date $T$. We convert the boundary value problem to an initial value problem. The first order conditions in (C.6) can be rewritten so that they imply a recursion for the optimal path. In particular, we have for $1 \leq t \leq T-1$ :

$$
u_{t+1}=g^{0}\left(u_{t}\right)+\frac{u_{t}-g^{0}\left(u_{t-1}\right)}{g_{x}^{0}\left(u_{t}\right)} .
$$

With an arbitrary $u_{1}$, we can then iterate on (C.16) until we hit a terminal $u_{T}$ which is at least $\epsilon$ away from $l^{*}$. Evaluating the rate function along this path, we obtain a value $S\left(T,\left(l^{*}, u_{1}, \ldots, u_{T}\right)\right)$. Then minimizing over the initial step $u_{1}$, we obtain the optimized rate function $\bar{V}$. This algorithm determines $T$ endogenously, and proved to be very fast in practice. Note that the entire algorithm must be carried out numerically, as (C.16) at each step uses the numerical policy function and its derivative.

\section{REFERENCES}

1. Amir, R. (1997) “A New Look at Optimal Growth Under Uncertainty," Journal of Economic Dynamics and Control, 22: 67-86. 
2. Araujo, A. (1991) "The Once But Not Twice Differentiability of the Policy Function," Econometrica, 59: 1383-1393.

3. Azencott, R. (1980) "Grandes Deviations et Applications," in Ecole d'Eté de Probabilités de Saint-Flour VIII-1978 Springer-Verlag, New York.

4. Blume, L., D. Easley, and M. O'Hara (1982) "Characterization of Optimal Plans for Stochastic Dynamic Programs," Journal of Economic Theory, 28: 221-234.

5. Brock, W.A. and L.J. Mirman (1972) "Optimal Economic Growth and Uncertainty: The Discounted Case", Journal of Economic Theory, 4: 479-513.

6. Campbell, J. (1994) "Inspecting the Mechanism: An Analytical Approach to the Stochastic Growth Model," Journal of Monetary Economics, 33: 463-506.

7. Dembo, A. and O. Zeitouni (1998) Large Deviations Techniques and Applications, Second Edition, Springer-Verlag, New York.

8. Dudley, R.M. (1989) Real Analysis and Probability, Wadsworth, Pacific Grove, California.

9. Fleming, W.H. (1971) "Stochastic Control for Small Noise Intensities," SIAM Journal on Control and Optimization, 9: 473-517.

10. Fleming, W.H. and M.R. James (1992) "Asymptotic Series and Exit Time Probabilities," Annals of Applied Probability, 20: 1369-1384.

11. Fleming, W.H. and P.E. Souganidis (1986) "Asymptotic Series and the Method of Vanishing Viscosity," Indiana University Mathematics Journal, 35: 425-447.

12. Freidlin, M.I. and A.D. Wentzell (1984) Random Perturbations of Dynamical Systems, Springer-Verlag, New York.

13. Gaspar, J. and K.L. Judd (1997) "Solving Large-Scale Rational Expectations Models," Macroeconomic Dynamics, 1: 45-75.

14. Johnson, N. and S. Kotz (1970) Continuous Univariate Distributions, Houghton Mifflin, Boston.

15. Judd, K.L. (1998) Numerical Methods in Economics, MIT Press, Cambridge. 
16. Judd, K.L. and S.M. Guu (1993) "Perturbation Solution Methods for Economic Growth Models," in Economic and Financial Modeling with Mathematica, (H. Varian, ed.) Springer-Verlag, New York.

17. Judd, K.L. and S.M. Guu (1997) "Asymptotic Methods for Aggregate Growth Models," Journal of Economic Dynamics and Control, 21: 1025-1042.

18. Kasa, K. (2001) "Learning, Large Deviations, and Recurrent Currency Crises," manuscript, Simon Fraser University.

19. Kim, J. and Kim, S. (2003) "Spurious Welfare Reversals in International Business Cycle Models," Journal of International Economics, 60: 471-500.

20. King, R.G., C.I. Plosser, and S.T. Rebelo (1988) "Production, Growth, and Business Cycles I: The Basic Neoclassical Model," Journal of Monetary Economics, 21: 309-342.

21. Klebaner, F.C. and R. Liptser (1999) "Moderate Deviations for Randomly Perturbed Dynamical Systems," Stochastic Processes and their Applications, 80: 157-176.

22. Klebaner, F.C. and O. Nerman (1994) "Autoregressive Approximation in Branching Processes with a Threshold," Stochastic Processes and their Applications, 51: 1-7.

23. Klebaner, F.C. and O. Zeitouni (1994) "The Exit Problem for a Class of Density-Dependent Branching Systems," Annals of Applied Probability, 4: 1188-1205.

24. Kushner, H.J. (1984) "Robustness and Approximation of Escape Times and Large Deviations Estimates for Systems with Small Noise Effects," SIAM Journal on Applied Mathematics, 44: 160-182.

25. Kushner, H.J. and G.G. Yin (1997) Stochastic Approximation Algorithms and Applications, SpringerVerlag, New York.

26. Kydland, F.E. and E.C. Prescott (1982) "Time to Build and Aggregate Fluctuations," Econometrica, 50: 1345-1370.

27. Lewis, F.L. (1986) Optimal Control, John Wiley \& Sons, New York.

28. Magill, M. (1977) "A Local Analysis of N-Sector Capital Accumulation Under Uncertainty," Journal of Economic Theory, 15: 211-218.

29. Prandini, J.C. (1994) "The Limiting Behavior of Solow's Model with Uncertainty when the Variance Goes to Zero," Economic Theory, 4: 799-809. 
30. Santos, M.S. (1993) "On High-Order Differentiability of the Policy Function," Economic Theory, 3: 565570.

31. Santos, M.S. (1991) "Smoothness of the Policy Function in Discrete Time Economic Models," Econometrica, 59: 1365-1382.

32. Santos, M.S. and J. Vigo-Aguiar (1998) "Analysis of a Numerical Dynamic Programming Algorithm Applied to Economic Models," Econometrica, 66: 409-426.

33. Stock, J. and M. Watson (1999) "Business Cycle Fluctuations in US Macroeconomic Time Series", in Handbook of Macroeconomics (J. Taylor and M. Woodford, eds.), Elsevier, Amsterdam.

34. Stokey, N.L. and R.E. Lucas, with E.C. Prescott (1989) Recursive Methods in Economic Dynamics, Harvard University Press, Cambridge. 